If you wish to distribute this article to others, you can order high-quality copies for your colleagues, clients, or customers by clicking here.

Permission to republish or repurpose articles or portions of articles can be obtained by following the guidelines here.

The following resources related to this article are available online at www.sciencemag.org (this infomation is current as of January 3, 2012 ):

Updated information and services, including high-resolution figures, can be found in the online version of this article at:

http://www.sciencemag.org/content/298/5598/1613.full.html

Supporting Online Material can be found at:

http://www.sciencemag.org/content/suppl/2002/11/20/298.5598.1613.DC1.html

A list of selected additional articles on the Science Web sites related to this article can be found at:

http://www.sciencemag.org/content/298/5598/1613.full.html\#related

This article cites 15 articles, 3 of which can be accessed free:

http://www.sciencemag.org/content/298/5598/1613.full.html\#ref-list-1

This article has been cited by 98 article(s) on the ISI Web of Science

This article has been cited by 16 articles hosted by HighWire Press; see:

http://www.sciencemag.org/content/298/5598/1613.full.html\#related-urls

This article appears in the following subject collections:

Evolution

http://www.sciencemag.org/cgi/collection/evolution 
0.08 and 0.10$)$ give estimated ages of $13,000 \pm 3,000$ and $17,000 \pm 3,000$ years for clades $\mathrm{B}$ and $\mathrm{C}$, respectively.

Thus, our mtDNA data suggest a first origin of domestic dogs either $\sim 40,000$ years ago, forming only clade $\mathrm{A}$, or $\sim 15,000$ years ago, possibly involving all the three clades A, B, and C. However, the oldest subcluster of clade A in Europe (as determined from the mean genetic distance from haplotypes unique to the western part of the world to the nodal haplotype shared with East Asia; 0.39 substitutions, $\mathrm{SD}=0.09$ ) is estimated to be only 9,000 $\pm 3,000$ years old (Fig. 2A). An origin of 40,000 years ago for clade A would therefore imply a long isolation in East Asia of dogs before they spread to the rest of the world. Circumstantial evidence therefore indicates a simultaneous origin in East Asia $\sim 15,000$ years ago for clades A and B, and possibly also clade $\mathrm{C}$.

In the context of the archaeological record, this seems to be a probable scenario. There is no certain evidence for domestic dogs in late Paleolithic China, but in the earliest Neolithic, finds are numerous, dating back to 7,500 $\mathrm{yr}$ B.P. $(4,19)$. Considering the relatively limited amount of archaeological work done in East Asia, the lack of late Paleolithic finds does not exclude a much earlier origin of domestic dogs in East Asia. The earliest Southwest Asian finds dated at $\sim 12,000 \mathrm{yr}$ B.P. are from unspecified small canids $(7,8)$, and remains with typical dog morphology appear only by 9,000 yr B.P. $(4,11)$. The German find from $14,000 \mathrm{yr}$ B.P. consists of a single jaw fragment (6), and there is a considerable temporal gap to later European finds, which appear by $\sim 9,000$ yr B.P. (4, 5, 12). The earliest North American finds are dated at 8,500 yr B.P. $(4,20)$. An East Asian origin is supported by a morphological feature of the jaw diagnostic of domestic dogs and also found in some Chinese wolves but generally not in other wolves $(4,10)$.

In conclusion, the archaeological record cannot define the number of geographical origins or their locations, but suggests the date at 9,000 to 14,000 yr B.P., while our mtDNA data indicate a single origin of domestic dogs in East Asia $\sim 15,000$ or 40,000 yr B.P. We conclude that a synthesis of available data points to an origin of the domestic dog in East Asia $\sim 15,000$ yr B.P. In this event, clade A would have had several origins from wolf haplotypes, and the first domestication of wolves would not have been an isolated event, but rather a common practice in the human population in question.

\section{References and Notes}

1. J. Clutton-Brock, in The Domestic Dog: Its Evolution Behaviour and Interactions with People, J. Serpell, Ed. (Cambridge Univ. Press, Cambridge, UK, 1995), PP 7-20.

2. C. Vila et al., Science 276, 1687 (1997)

3. R. K. Wayne, Trends Genet. 9, 218 (1993).
4. S. J. Olsen, Origins of the Domestic Dog (University of Arizona Press, Tucson, 1985).

5. N. Benecke, J. Archaeol. Sci. 14, 31 (1987).

6. G. Nobis, Umschau 79, 610 (1979).

7. S. J. M. Davis, F. R. Valla, Nature 276, 608 (1978).

8. E. Tcherncov, F. R. Valla, J. Archaeol. Sci. 24, 65 (1997).

9. J. Clutton-Brock, A Natural History of Domesticated Mammals (Cambridge Univ. Press, Cambridge, UK, ed. 2, 1999).

10. S. J. Olsen, J. W. Olsen, Science 197, 533 (1977).

11. B. Lawrence, Z. Saugertierkd. 32, 44 (1967).

12. M. Degerböl, Proc. Prehist. Soc. 27, 35 (1961).

13. Dogs were categorized into geographic groups on the basis that they either were of a breed with known geographic origin or were living in an area with little import of foreign breeds: Africa (sample size $n=35$ ) [North $(n=8)$, South $(n=27)$ of Sahara]; America $(n=$ $25)$; Europe $(n=207)$ [British Isles $(n=65)$, Continent $(n=93)$, Scandinavia $(n=49)]$; East Asia $(n=260)$ [China $(n=129)$, Japan $(n=96)$, Southeast Asia $(n=$ 21), miscellaneous ( $n=14)$ ]; Southwest Asia, here defined as Asia west of China and India $(n=90)$ [Turkey $(n=24)$, Israel $(n=18)$, Iran $(n=14)$, miscellaneous $(n=34)]$; India $(n=13)$.

14. Materials and methods are available on Science Online.

15. N. Okumura, N. Ishiguro, M. Nakano, A. Matsui, M. Sahara, Anim. Genet. 27, 397 (1996).

16. C. Vila et al., Mol. Ecol. 8, 2089 (1999).

17. B. Kurtén, E. Anderson, Pleistocene Mammals of North America (Columbia Univ. Press, New York, 1980).

18. Y.-X. Fu, Genetics 147, 915 (1997).

19. Q. Li, Chin. J. Cynol. 2, 25 (1990).

20. J. Clutton-Brock, N. Noe-Nygaard, J. Archaeol. Sci. 17, 643 (1990).

21. D. Posada. Modeltest 3.06 (Brigham Young University, Provo, UT, 2001)

22. D. L. Swofford, PAUP* 4.0b10 (Sinauer Associates, Sunderland, MA, 2002).

23. This work was supported by the Swedish Research Council, the Swedish Kennel Club, the State Key Basic Research and Development Plan of China (G20000161), and Natural Sciences Foundation of China. We thank numerous contributors of samples, especially J. KolerMatznick and D. Selin for sampling coordination and L. Arvestad for help with calculations. mtDNA sequences have been deposited in GenBank (accession numbers AF530561 to AF530566 and AF531654 to AF531741).

Supporting Online Material

www.sciencemag.org/cgi/content/full/298/5598/1610/ DC1

Material and Methods

Figs. S1 to S3

Tables S1 to S3

13 May 2002; accepted 25 September 2002

\title{
Ancient DNA Evidence for Old World Origin of New World Dogs
}

\author{
Jennifer A. Leonard, ${ }^{1 *} \dagger$ Robert K. Wayne, ${ }^{1}$ Jane Wheeler, ${ }^{2}$ \\ Raúl Valadez, ${ }^{3}$ Sonia Guillén, ${ }^{4}$ Carles Vilà ${ }^{5}$
}

\begin{abstract}
Mitochondrial DNA sequences isolated from ancient dog remains from Latin America and Alaska showed that native American dogs originated from multiple Old World lineages of dogs that accompanied late Pleistocene humans across the Bering Strait. One clade of dog sequences was unique to the New World, which is consistent with a period of geographic isolation. This unique clade was absent from a large sample of modern dogs, which implies that European colonists systematically discouraged the breeding of native American dogs.
\end{abstract}

The dog is the only domesticated species that was distributed across Eurasia and the Americas before the development of transoceanic travel during the 15 th century. Genetic, morphologic, and behavioral studies (1-5) have

1Department of Organismic Biology, Ecology and Evolution, University of California, Los Angeles, CA 90095-1606, USA. ${ }^{2}$ CONOPA, Coordinadora de Investigación y Desarrollo de Camélidos, Avenida Fernando Reusche, Mz. M, Lote 4, Pachacamac, Lima 19, Peru. ${ }^{3}$ Laboratorio de Paleozoología, Instituto de Investigaciones Antropológicas de la Universidad Nacional Autónoma de México, Circuito Exterior, s/n, Ciudad Universitaria, Delegación Coyoacán, C.P. 04510, México D.F., Mexico. ${ }^{4}$ Centro Mallqui, The Bioanthropology Foundation Peru, Avenida A. Márquez 2014, Jesús María - Lima 11, Peru. ${ }^{5}$ Department of Evolutionary Biology, Uppsala University, Norbyvägen 18D, S-75236 Uppsala, Sweden.

*To whom correspondence should be addressed. Email: Leonard.Jennifer@NMNH.SI.edu.

$\dagger$ Present address: Genetics Program, Department of Systematic Biology, National Museum of Natural History, Smithsonian Institution, 3001 Connecticut Avenue N.W., Washington, DC 20008-0551, USA. shown that domestic dogs derive from the gray wolf (Canis lupus), a species that has a holarctic distribution. Therefore, domestic dogs were either brought to the New World by late Pleistocene humans crossing the Bering Strait from Asia or were domesticated in the New World independently from gray wolves. A New World domestication of dogs is supported by morphological ( 1 ) and limited genetic data (6). Further, the antiquity of dog and human remains in the New World suggests independent Old and New World domestication events. The oldest dog remains in the New World, from Danger Cave, Utah (7), are dated from 9000 to 10,000 years before the present (yr B.P.) and compare with the earliest dates of about 12,000 to $14,000 \mathrm{yr}$ B.P. for dog remains from archaeological sites in Germany, Israel, and Iraq $(8-10)$. The arrival of humans in North America occurred by at least 12,000 to 14,000 yr B.P. $(11,12)$ and therefore was coincident with or predated the first appearance of dogs in the archaeo- 


\section{REPOR T S}

logical record. Consequently, native American dogs are likely to have derived from American wolves.

Modern New World dogs are of questionable use in determining their origin, because native American dogs likely interbred with dogs brought by European colonists and ultimately may have been replaced by them (13, 14). Consequently, we extracted DNA from bones of $37 \mathrm{dog}$ specimens from archaeological sites in Mexico, Peru, and Bolivia that were deposited before the arrival of Columbus in the New World (15). We have successfully amplified 425 base pairs (bp) of the mitochondrial DNA (mtDNA) control region from 13 of them (Table 1). Further, we have analyzed sequences from $11 \mathrm{dog}$ remains from Alaska, deposited before the first arrival of European explorers (15) (Table 1). We found 12 different haplotypes in 13 samples from pre-Columbian dogs from Latin America. The two identical sequences originated from the same locality [JAL 332 and JAL 334 from Bolivia (Table 1)]. The haplotypes differed by 1 to $12 \mathrm{bp}(0.2$ to $3.1 \%$ divergence). To compare our sequences to those from previous studies, we focused on a 257 bp fragment of the control region that was homologous to sequences from 140 dogs obtained from 67 diverse dog breeds (5) and to sequences from 259 wolves obtained from 30 localities worldwide (16). When just this fragment was considered, the ancient Latin American sequences defined 11 haplotypes. Twenty-five additional homologous dog sequences were found in GenBank, which yielded five new haplotypes. Almost all modern dogs were sampled in the Old World, and most corresponded to breeds originating there. The few sequences from breeds of New World origin (the Eskimo dog, Mexican hairless, Alaskan husky, Newfoundland, and Chesapeake Bay retriever) and from Oceania (the Australian dingo and the New Guinea singing dog) were indistinguishable from those of Eurasian dogs (5).

A phylogenetic tree based on the 257-bp sequences (15) (Fig. 1) showed that the ancient American dog sequences clustered within two of the four previously defined dog clades (5). Ten of the pre-Columbian American dog haplotypes were clustered in clade I, and one sequence from Tula, Mexico [PC 13 (Table 1)], was clustered in clade IV. Clade I is the most diverse clade of dog sequences and comprises about $80 \%$ of dog haplotypes, including the Australian dingo, the New Guinea singing dog, the African basenji, the greyhound, and other ancient breeds. American gray wolf sequences (Fig. 1, lu 28 to lu33 in blue) are not clustered with those from dogs and differ by 3 to $13 \mathrm{bp}$ from the ancient American dog sequences. Three of the ancient sequences are identical to those observed in Eurasian dogs, and none differs by more than 5 bp (Fig. 1). However, when the sequence of the entire 425-bp segment from ancient samples is compared to over 350 modern dog sequences of different lengths (17), only the pre-Columbian sequence from sample PC13 is identical to one found in modern dogs (haplotype D6). These results suggest that New and Old World dogs are derived from Eurasian wolves.

Our pre-Columbian samples were from Latin America, where gray wolves are rare or absent. Consequently, we obtained remains from the permafrost deposits of Alaska, where gray wolves were abundant in the past and could provide a source for domestication and interbreeding (1). These deposits often yield relatively well-preserved DNA from specimens as old as 50,000 years (18). However, the $11 \mathrm{dog}$ remains that we tested dated between 1450 and 1675 C.E., and thus most postdate the first arrival of European colonists in the New World (Table 1). Nevertheless, all of these dog remains were deposited before the first sighting of Alaska by Europeans (by Vitus Bering and Aleksey Chirikov in 1741) and thus should represent pure native American dogs. Based on the 257-bp sequence, we found 8 haplotypes in 11 samples. Five are unique, whereas three are shared with modern domestic dogs [D1, D3, and D18 (Fig. 1)]. All ancient Alaskan dog sequences possess clade I haplotypes (Fig. 1, green).

To better visualize the relationship of modern and ancient dog sequences from clade I, we created a statistical parsimony network where haplotypes can occupy nodes and where each branch represents a single nucleotide substitution or an insertion or deletion (15) (Fig. 2). This network shows that haplotype D28, found in ancient samples from Bolivia, is ancestral to a clade of unique New World haplotypes and differs from them by one or two substitutions. Haplotypes D36, found in ancient Alaskan dogs, or D2, a common haplotype in Old World dogs, are putatively ancestral to this endemic New World clade that we designate as clade $a$ (Fig. 2 ). The statistical parsimony network suggests that the remaining ancient sequences might be derived from the common haplotypes D2, D3, D9, and D26 (Fig. 2) (5). Further, an additional lineage must be derived from the clade IV haplotype D6 (Fig.

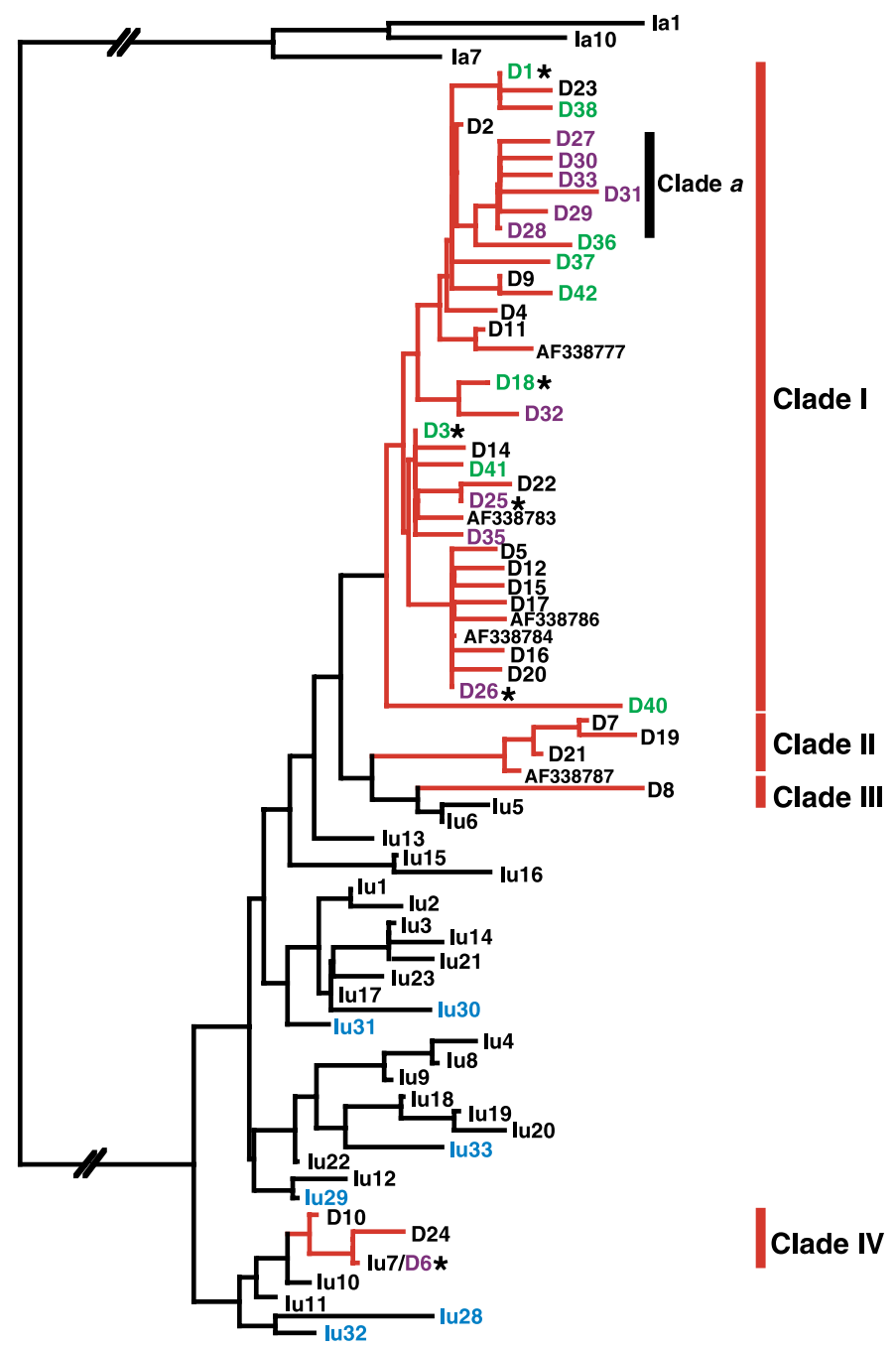

Fig. 1. Neighbor-joining tree of sequences from precontact dogs from Latin America (purple) and Alaska (green) and modern dogs [black, prefix $D$, for sequences from (5), or GenBank accession numbers], Eurasian wolves [black, prefix lu, from (16)], and American wolves [blue, prefix lu, from (16)]. Coyotes [black, prefix la, from (16)] are used as an outgroup. Previously defined clades of dogs (5) are indicated with red branches. Clade a refers to a group of dog sequences unique to the New World. Haplotypes shared between modern and ancient dogs are marked with an asterisk. 


\section{R E P O R T S}

1). Consequently, our phylogenetic analysis suggests that minimally, about five founding dog lineages (including the ancestor of clade $a$ ) invaded North America with humans as they colonized the New World. Further, the presence of the unique haplotype group (clade $a$ ) derived from New World haplotype D28 is consistent with a history of isolation (19).
Analysis of ancient sequences from New World dog remains from localities as distant as Peru and Alaska supports the hypothesis that ancient and modern dogs worldwide wolves. Our results differ from those of a previous study (6) that reported sequences closely related to New World wolves in four
Fig. 2. Statistical parsimony cladogram of precontact Latin American (gray), Alaskan (black), types from clade I (5). Modern sequences from (5) are labeled $D$, and other sequences from GenBank are labeled with their accession number. Each branch represents a 1-bp change or indel, and dots represent hypothetical haplotypes.

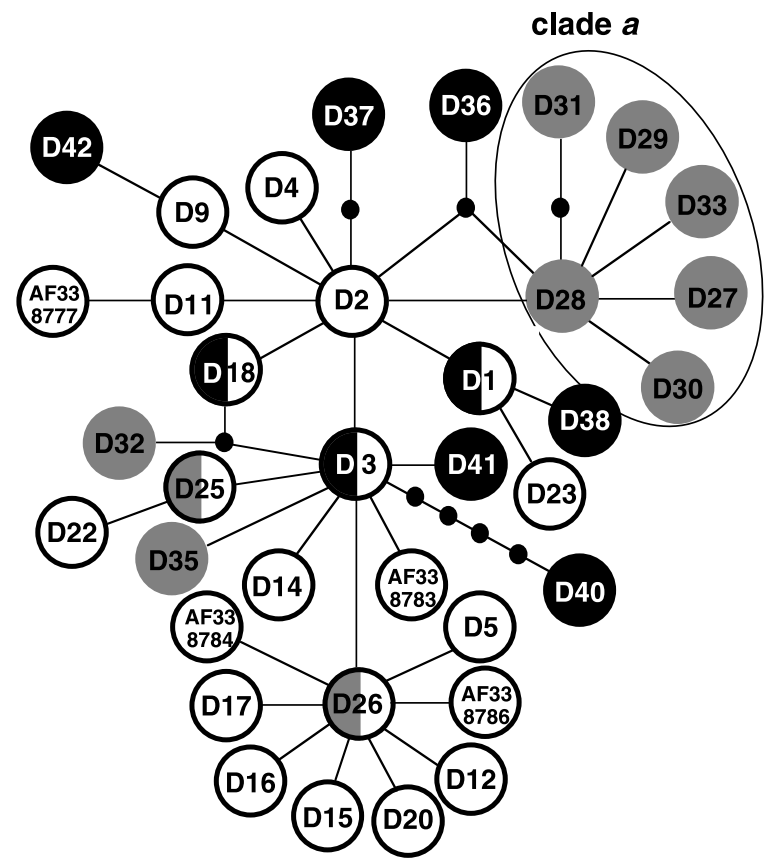
share a common origin from Old World gray and modern (white) dog haplo-

of five dog remains associated with native Americans in British Columbia. This finding could represent localized interbreeding between domestic dogs and North American wolves, as suggested by morphologic $(20,21)$ and historic $(13,21)$ data. However, our data suggest that widespread introgression of female wolf matrilines into the native dog population occurred infrequently, because all sequences from ancient native dogs were well differentiated from those found in North American wolves. Similarly, extant North American gray wolves show no genetic evidence of interbreeding with dogs, despite the high concentration of dogs in many areas occupied by gray wolves (22). Hybridization is occasionally observed in Europe, where dogs are common and gray wolves are rare (23).

Six of 12 ancient Latin American haplotypes are grouped in clade $a$ and include sequences found in dog remains from Bolivia, Peru, and Mexico (Fig. 1). No sequences from clade $a$ have been found in samples from over 350 modern dogs (17). The upper bound of a $95 \%$ confidence limit for the frequency that sequences from clade $a$ could have in this modern sample and be missed (an observed frequency of zero) is $1.0 \%$ (24). Consequently, the absence of clade $a$ sequences from modern dogs suggests an extensive replacement of native American dogs by those introduced by Europeans. These lin-

Table 1. Sample source, locality, age, and haplotype, based on a 257-bp segment of the mitochondrial control region (5). Haplotypes D1, D3, D6, D18, D25, and D26, shown in bold, have previously been reported (5), and all other haplotypes are novel. F:AM samples are from the American Museum of

Natural History, New York. The ages for Alaskan samples are radiocarbon dates from the NSF-Arizona Accelerator, Mass Spectrometry Facility. RYBP, radiocarbon years before the present; C.E., calendar year (common era). Radiocarbon years were converted to calendar years using the tables in (29).

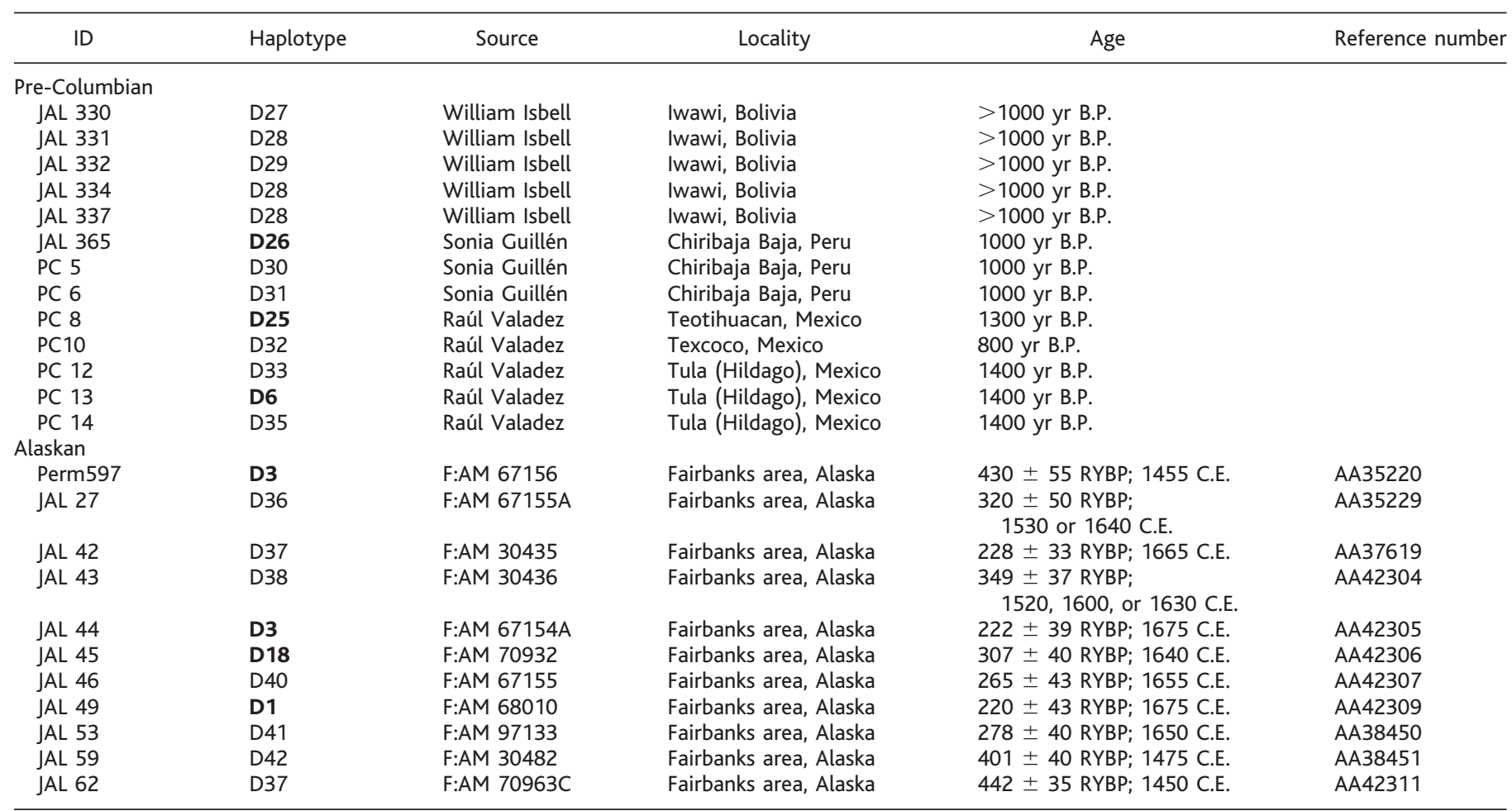


eages could be surviving in some unsurveyed modern Native American breeds or local dog populations $(14,25)$. However, genetic analysis of a diverse sample of 19 Mexican hairless dogs (xoloitzcunitle), a distinct ancient breed that has been present in Mexico for over 2000 years (25), only revealed mtDNA sequences previously observed in dogs of Eurasian origin (26). The absence of ancient North and South American dog haplotypes from a large diversity of modern breeds, including the Mexican hairless, illustrates the considerable impact that invading Europeans had on native cultures.

Our data strongly support the hypothesis that ancient American and Eurasian domestic dogs share a common origin from Old World gray wolves. This implies that the humans who colonized America 12,000 to $14,000 \mathrm{yr}$ B.P. brought multiple lineages of domesticated dogs with them. The large diversity of mtDNA lineages in the dogs that colonized the New World implies that the ancestral population of dogs in Eurasia was large and well mixed at that time. Consequently, dogs, in association with humans or through trade, spread across Europe, Asia, and the New World soon after they were domesticated. Alternatively, if domestication was a more ancient event, as suggested by previous genetic results (5), human groups that first colonized the subarctic mammoth steppe of Siberia may have had dogs with them 26,000 to $19,000 \mathrm{yr}$ B.P. (11). If the archaeological date of 12,000 to $14,000 \mathrm{yr}$ B.P. for first domestication is accepted, the dog, as an element of culture, would have had to be transmitted across Paleolithic societies on three continents in a few thousand years or less. This would imply extensive intercultural exchange during the Paleolithic $(27,28)$. Regardless, the common origin of New and Old World dogs demands a reconsideration of the relationship between humans and dogs in ancient societies.

References and Notes

1. S. J. Olsen, Origins of the Domestic Dog: The Fossil Record (Univ. of Arizona Press, Tucson, AZ, 1985).

2. D. F. Morey, Am. Sci. 82, 336 (1994).

3. J. P. Scott, J. L. Fuller, Dog Behavior: The Genetic Basis (Univ. of Chicago Press, Chicago, IL, 1965)

4. R. K. Wayne, S. J. O'Brien, Syst. Zool. 36, 457 (1987).

5. C. Vilà et al., Science 276, 1687 (1997).

6. B. F. Koop, M. Burbidge, A. Byun, U. Rink, S. J. Crockford, in Dogs Through Time: An Archaeological Perspective, S. J. Crockford, Ed. (British Archaeological Reports, Oxford, 2000), pp. 271-285. Two of the five ancient remains studied were dated to 1938 and 1940; the rest were designated as "prehistoric" by the authors.

7. D. K. Grayson, Anthropol. Pap. Am. Mus. Nat. Hist. 66 (1988).

8. G. Nobis, Umschau 19, 610 (1979).

9. T. Dayan, J. Archaeol. Sci. 21, 633 (1994).

10. J. Clutton-Brock, A Natural History of Domesticated Mammals (Cambridge Univ. Press, Cambridge, ed. 2, 1999).

11. T. Goebel, Evol. Anthropol. 8, 208 (1999).

12. S. J. Fiedel, J. Archaeol. Res. 8, 39 (2000).
13. M. Schwartz, A History of Dogs in the Early Americas (Yale Univ. Press, New Haven, CT, 1997).

14. R. Valadez, G. Mestre, Historia del Xoloitzcuintle en México (Museo Dolores Olmedo-UNAM, México, 1999).

15. Supporting material is available on Science Online.

16. C. Vilà et al., Mol. Ecol. 8, 2089 (1999).

17. The 350 modern dog sequences are from over 250 dogs corresponding to 124 haplotypes with sequences deposited in GenBank, and 100 dogs from 20 different breeds (C. Vilà, data not shown).

18. I. Barnes, P. Matheus, B. Shapiro, D. Jensen, A. Cooper, Science 295, 2267 (2002).

19. J. C. Avise, Phylogeography. The History and Formation of Species (Harvard Univ. Press, Cambridge, MA, 2000).

20. J. Clutton-Brock, A. C. Kitchener, J. M. Lynch, J. Zool. 233, 19 (1994)

21. R. Valadez, et al. Asoci. Mexi. Med. Vet. Especi. Pequeñas Especies 13, 6 (2002).

22. C. Vilà, R. K. Wayne, Conserv. Biol. 13, 195 (1999).

23. E. Randi, V. Lucchini, Conserv. Genet. 3, 31 (2002).

24. J. H. Zarr, Biostatistical Analysis (Prentice Hall, Upper Saddle River, NJ, ed. 4, 1999); the calculation is made according to example 24.5.

25. R. Valadez, in Dogs Through Time: An Archaeological
Perspective, S. J. Crockford, Ed. (British Archaeological Reports, Oxford, 2000), pp. 193-204.

26. C. Vilà, J. E. Maldonado, R. K. Wayne, J. Hered. 90, 71 (1999).

27. C. Gamble, The Palaeolithic Societies of Europe (Cambridge Univ. Press, Cambridge, 1999).

28. A. P. Derev'anko, in The Paleolithic of Siberia, New Discoveries and Interpretations, A. P. Derev'anko, D. B. Shimkin, W. R. Powers, Eds. (Univ. of Illinois Press, Chicago, IL, 1998), pp. 5-13.

29. M. Stuiver, G. W. Pearson, Radiocarbon 35, 1 (1993).

30. We thank R. Tedford (American Museum of Natural History, New York) and W. Isbell (Department of Anthropology, State University of New York at Binghamton) for samples. This research was supported by grants from the University of California Institute for Mexico and the United States and NSF (grant OPP9817937). We thank C. Anderung, J. Brantingham, A. Götherström, B. VanValkenburgh, and M. Zeder for comments on the manuscript.

\section{Supporting Online Material}

www.sciencemag.org/cgi/content/full/298/5598/1613/

DC1

Materials and Methods

References

5 August 2002; accepted 26 September 2002

\title{
Whole-Genome Analysis of Photosynthetic Prokaryotes
}

\section{Jason Raymond, ${ }^{1 *}$ Olga Zhaxybayeva, ${ }^{2 *}$ J. Peter Gogarten, ${ }^{2}$ Sveta Y. Gerdes, ${ }^{3}$ Robert E. Blankenship ${ }^{1} \dagger$}

\begin{abstract}
The process of photosynthesis has had profound global-scale effects on Earth; however, its origin and evolution remain enigmatic. Here we report a wholegenome comparison of representatives from all five groups of photosynthetic prokaryotes and show that horizontal gene transfer has been pivotal in their evolution. Excluding a small number of orthologs that show congruent phylogenies, the genomes of these organisms represent mosaics of genes with very different evolutionary histories. We have also analyzed a subset of "photosynthesis-specific" genes that were elucidated through a differential genome comparison. Our results explain incoherencies in previous data-limited phylogenetic analyses of phototrophic bacteria and indicate that the core components of photosynthesis have been subject to lateral transfer.
\end{abstract}

Photosynthesis is an essential biological process in which solar energy is transduced into other forms of energy that are available to all life. Primary production by photosynthetic organisms supports all ecosystems, with the noted exceptions of deep-sea hydrothermal vents and subsurface communities. Oxygen, one of the by-products of photosynthesis by cyanobacteria and their descendants (including algae and higher plants), transformed the Precambrian Earth and made possible the development of more complex organisms that use aerobic metabolism $(1,2)$. Understanding the origin and evolution of the process of photosyn-

${ }^{1}$ Department of Chemistry and Biochemistry, Arizona State University (ASU), Tempe, AZ 85287-1604, USA. 2Department of Molecular and Cell Biology, University of Connecticut, Storrs, CT 06269-3044, USA. ${ }^{3}$ Integrated Genomics, 2201 West Campbell Park Drive, Chicago, IL 60612, USA.

*These authors contributed equally to this paper. $\dagger$ To whom correspondence should be addressed. Email: blankenship@asu.edu thesis is, therefore, of considerable interest.

All available evidence suggests that (bacterio)chlorophyll-based photosynthesis arose within the bacterial domain of the tree of life and was followed by subsequent endosymbiotic transfer into eukaryotes. Accurate dates for appearance of the first photosynthetic organisms are not known. Substantial information, including biomarkers, stromatolites, and paleosols, as well as data from molecular evolution studies, indicates that oxygenic (oxygen-evolving) photosynthesis arose by 2500 million years ago (2-5). On the basis of phylogenetic analyses and the well-detailed complexity of the photosynthetic machinery, mechanistically simpler anoxygenic (nonoxygen-evolving) photosynthesis almost certainly preceded and was ancestral to oxygenic photosynthesis $(1,6)$. Therefore the cyanobacteria, as ancient as they appear to be, were probably preceded by a diverse group of more primitive phototrophs. The supposed progeny of those early phototrophs are still 


\section{Supporting Online Material}

A 425bp fragment of the mitochondrial DNA control region was amplified in three overlapping fragments from bones, teeth or mummified soft tissue of ancient American dogs (primers Thr-L 5'-GAA TTC CCC GGT CTT GTA AAC C and dogDL-5 5'-CAT TAA TGC ACG ACG TAC ATA GG; dogDL-1g 5'GTG CTA TGT CAG TAT CTC CAG G and dogDL-2 5'GCA AGG GTT GAT GGT TTC TCG or dogDL-3 5'-CCC TTA TTG GAC TAA GTG ATA TGC AT; dogDL-4 5'-GCA TAT CAC TTA GTC CAA TAA GGG or dogDL-7 5'-TAT TAT ATC CTT ACA TAG GAC and DL-Hcan 5'-CCT GAG GTA AGA ACC AGA TG). Polymerase chain reaction and extraction conditions as well as precautions applied to the study of ancient DNA are as described in ref. 1 . Thirty-seven specimens from archaeological sites in Mexico ( $\mathrm{N}=$ 6), Peru ( $\mathrm{N}=26)$ and Bolivia $(\mathrm{N}=5)$ and dated by their archaeological context as pre-Columbian were analyzed. Complete sequences were obtained from 13 of these specimens. To confirm reliability of the sequences obtained, at least one fragment was replicated for each sample, three samples were extracted and sequenced two or three times (at UCLA by JAL) and one sequence fragment was independently extracted, amplified and sequenced with separate reagents at the University of Uppsala by CV. Sequences have been deposited into GenBank.

Phylogenetic trees were built using a neighbor-joining algorithm and a HKY85 model of evolution assuming variation in the rate of nucleotide substitutions across loci following a gamma distribution with parameter $a=0.5$. Neighbor-joining trees were constructed assuming other models of sequence evolution, as well as trees using different optimality criteria (maximum parsimony and maximum likelihood). Trees were build using PAUP*4.10b (2). In all cases, the same framework topology was observed, with four well-differentiated groups of dog sequences as 
in ref. 3. See ref. 3 for analysis of statistical support for the four dog clades. A clade of Americanspecific sequences (clade $a$ ) was retained in all trees.

When ancestral haplotypes are likely to be present in a population, such as in dogs, networks may be a more efficient way to represent phylogenetic relationships since they allow for sequences at the internal nodes. For dog sequences in Clade I (Fig. 1) we have constructed a statistical parsimony network with the program TCS v1.13 (4), where all haplotypes are connected by branches representing single mutational events, and insertions/deletions were used as the fifth character state.

\section{References}

1. J. A. Leonard, R. K. Wayne, A. Cooper, Proc. Natl. Acad. Sci. 97,1651 (2000).

2. D. L. Swofford, PAUP*. Phylogenetic Analysis Using Parsimony (*and Other Methods). Version 4. (Sinauer Associates, Sunderland, Massachusetts, 1998).

3. C. Vilà, et al. Science 276,1687 (1997).

4. M. Clement, D. Posada, K. A. Crandall, Mol. Ecol. 9, 1657 (2000). 